SHORT REPORT

\title{
Coexistent blepharospasm and hemifacial spasm: overlapping pathophysiologic mechanism?
}

\author{
E-K Tan, L-L Chan, K-K Koh
}

J Neurol Neurosurg Psychiatry 2004;75:494-496. doi: 10.1136/jnnp.2003.019331

\begin{abstract}
Background/aim: Blepharospasm (BEB) and hemifacial spasm (HFS) appear to be distinct disorders. Clinical characteristics of coexistent BEB and HFS have not been examined. The aim of this study was to determine the prevalence, clinical, and imaging features of coexistent BEB among a cohort of HFS patients and controls.

Results: Among 665 study subjects, nine (5.5\%) of the 164 consecutive HFS patients had coexistent BEB, significantly higher than age and gender matched controls $(0 / 501,0 \%)$ without neurological diseases $(p<0.0001)$. The mean age of the nine patients was 61.4 (SD 9.9) (range 51-72), consisting of $88.9 \%$ women, and $66.7 \%$ had left sided HFS, similar to HFS patients without BEB. Six (66.7\%) reported $B E B$ symptoms at a mean of 0.8 years after HFS onset, one before, and onset was undetermined in two patients. Advanced magnetic resonance imaging and angiography revealed neurovascular compression of the ipsilateral side of HFS, without any basal ganglia lesions.

Conclusions: BEB occurred more frequently in HFS patients, suggesting changes in the brainstem blink reflex circuitry could play a modulatory role in certain at-risk individuals resulting in the coexistence of these movement disorders.
\end{abstract}

$\mathrm{H}$ emifacial spasm (HFS) is characterised by clonic or tonic contractions of muscles innervated by the ipsilateral facial nerve. ${ }^{1-3}$ Vascular compression at the root exit zone of the facial nerve is the commonest underlying aetiology of HFS. ${ }^{3-5}$ Electrophysiological studies suggest the presence of ephaptic transmission and facial nucleus hyperactivity. ${ }^{6}$ Blepharospasm (BEB) consists of bilateral, usually symmetrical, and synchronous contractions of the orbicularis oculi, frequently also involving the frontalis and corrugator muscles. ${ }^{8}$ Primary BEB has no known aetiology, whereas secondary BEB is associated with lesions in the basal ganglia, brainstem, and thalamus. ${ }^{10-14}$ Dystonia of the lower face and jaw, and cervical dystonia are frequently associated with BEB. ${ }^{8}{ }^{9}$ Being female, a family history of dystonia or postural tremor, prior eye disease, and dental procedures are risk factors. ${ }^{10-14}$ In some cases there may be an increased genetic susceptibility..$^{10} 14$

We hypothesise that both BEB and HFS can coexist, sharing overlapping pathophysiologic mechanisms. In a case control study, we determined the prevalence, clinical, and advanced magnetic resonance imaging and angiography (MRI/A) features of coexistent BEB in a cohort of HFS patients and controls.

\section{METHODS}

We included 164 consecutive HFS patients in the botulinum toxin clinic and 501 outpatient controls without neurological disorders (for every patient, we examined about three age and sex matched controls). HFS patients who satisfied all the following criteria were diagnosed to have coexistent HFS and BEB: (1) a clinical diagnosis of HFS manifested by involuntary tonic and/or clonic contractions of the muscles innervated by the ipsilateral facial nerve; (2) a clinical diagnosis of BEB characterised by frequent blinking which progressed to sustained tonic contraction of both the orbicularis oculi, and (3) separate occurrence of HFS and BEB episodes. The exclusion criteria were patients with facial tics, dystonia, hemimasticatory spasm, tardive dyskinesia, other forms of facial or oromandibular dystonic movements, and bilateral HFS. Patients were investigated with advanced MRI/A imaging techniques sensitive for neurovascular compression. Controls who satisfied a clinical diagnosis of BEB based on history and examination were determined. $\chi^{2}$ test and student $t$ test were used to compare the categorical and continuous variables between patients and controls.

\section{RESULTS}

Of the 665 study subjects, there were 164 HFS patients with mean age of 54.8 (SD 12.1) (range 27-80) years, and (102/ 164) $62.2 \%$ of them women, and 501 controls without neurological diseases with mean age of 51.4 (SD 14.4) (range $32-90)$ years, and (306/501) 61.1\% were women. Nine $(5.5 \%)$ of 164 consecutive HFS patients diagnosed in the outpatient clinic had coexistent BEB, significantly higher than the outpatient controls $(0 / 501,0 \%)(p<0.0001)$. The mean age of the nine patients was 61.4 (SD 9.9) (range 51-72), consisting of $88.9 \%$ women, and $66.7 \%$ had left sided HFS, similar to HFS patients without BEB. Six (66.7\%) reported BEB symptoms at a mean of 0.8 years after HFS onset, one before, and in two patients the timing cannot be accurately determined (table 1). MRI/A revealed neurovascular compression of the ipsilateral side of HFS, without any basal ganglia lesions in five patients (table 1). No abnormality was observed in one patient with Bell's palsy associated HFS and BEB. MRI/A were not available for analysis in three patients.

\section{ILLUSTRATIVE CASE REPORTS \\ Patient 8}

The patient, a 60 year old woman, complained of twitching in the left lower lid followed by the left lower facial muscles, resulting in eyelid closure and pulling of angle of her mouth upwards. She was diagnosed to have left HFS and treated with botulinum toxin with improvement. Less than a year later, she noticed twitching of her right and left eyelids simultaneously, accompanied by frequent eye blinking and eyelid closure. These movements worsened under bright daylight. This was separate from the episodes of her left sided HFS. MRI/A examination revealed neurovascular compression of the ipsilateral facial nerve by the left posterior inferior

Abbreviations: BEB, blapharospasm; HFS, hemifacial spasm; MRI/A, magnetic resonance imaging/angiography 
Table 1 Patients with coexistent hemifacial spasm and blepharospasm

\begin{tabular}{|c|c|c|c|c|}
\hline Patient (age, sex) & $\begin{array}{l}\text { Hemifacial spasm } \\
\text { (duration, side) }\end{array}$ & $\begin{array}{l}\text { Blepharospasm (onset } \\
\text { before/after) }\end{array}$ & MRI/A findings & Associated diseases \\
\hline 1. 56 , female & $7 \mathrm{yr}$, left & 1 yr after & Left VA & Hypertension \\
\hline 2. 52 , female & $2.5 \mathrm{yrs}$, right & $0.5 \mathrm{yr}$ after & Normal & Left Bell's palsy \\
\hline 3. 72 , female & 2 yrs, left & 2 yr before & Left AICA & $\begin{array}{l}\text { Diabetes, heart } \\
\text { disease, head tremor }\end{array}$ \\
\hline 4. 76 , female & 4 yrs, right & 1 yr after & Not available & Nil \\
\hline 5. 58 , male & $1 \mathrm{yr}$, left & 1 yr after & Not available & Hand tremor \\
\hline 6. $51 /$ Female & $2.5 y r s$, right & Undetermined & Right PICA & Nil \\
\hline 7. 54 , female & 1.5yrs, left & $0.1 \mathrm{yr}$ after & Left PICA & $\begin{array}{l}\text { Past history of thyroid } \\
\text { disease }\end{array}$ \\
\hline 8. 60 , female & 6 yrs, left & 1 yr after & Right PICA & Hypertension \\
\hline 9. 75 , female & $2.5 y r s$, left & Undetermined & Not available & $\mathrm{Nil}$ \\
\hline
\end{tabular}

cerebeller artery. All her symptoms were effectively treated with botulinum toxin.

\section{Patient 1}

This patient, a 52 year old woman, had mild residual right facial weakness after suffering from a right Bell's palsy. About two years later, she developed twitching of her right lower eyelid, and two months later, her right lower facial and platysma muscles were also involved. She was diagnosed to have right HFS. An MRI/A examination did not reveal any significant neurovascular compression of the ipsilateral facial nerve. One year after the HFS onset, she started complaining of frequent blinking followed by twitching and spasms of both right and left eyelids. The eyelid contractions were bilaterally synchronous, separate from the episodes of her right HFS. Both HFS and BEB responded to botulinum toxin injections.

\section{DISCUSSION}

We found a higher prevalence of BEB in a cohort of HFS compared with age and sex matched controls $(5.5 \% \vee 0 \%$, $\mathrm{p}<0.0001$ ). The likelihood of selection bias or coincidence of BEB among HFS patients was small because BEB was rarely encountered than HFS in our clinic population (estimated to be around $1: 15)$, and the majority $(66.7 \%)$ of the nine patients developed BEB after HFS onset. The clinical characteristics of HFS patients with and without BEB did not differ. BEB was not reported to occur in an American HFS study sample. ${ }^{2}$ Conversely, studies of BEB patients in white populations frequently described coexistent craniocervical dystonia and essential tremor but not HFS. ${ }^{8}{ }^{9}$ We do not know whether there are any ethnicity specific differences in these observations, as HFS is relatively rare among whites.

Other differential diagnosis such as bilateral HFS was unlikely, as advanced MRI/A imaging demonstrated neurovascular compression of the ipsilateral facial nerve in the majority of our cases, and there were no structural lesions to account for both HFS and BEB. All the patients had simultaneous onset of their BEB symptoms bilaterally (separate from their ipsilateral HFS symptoms), unlike the long latency of onset between the two sides reported in bilateral HFS.$^{4}$ In three patients, family members had noticed the ipsilateral facial spasm but not BEB movements during sleep. Persistent HFS symptoms during sleep are well recognised. ${ }^{2}$ Furthermore, patients with cranial dystonic movements such as Meige syndrome and tardive dystonia/ dyskinesia were excluded.

BEB and HFS appear to be distinct disorders with differing patho-aetiologies. While structural lesions in basal ganglia and other risk factors have been described in BEB, most cases are of unknown aetiology. ${ }^{8}$ On the other hand, neurovas- cular compression of the root exit zone of the ipsilateral facial nerve is the most common cause of HFS. ${ }^{1-5}$ Current evidence suggests that genetic predisposition is more important than environmental factors in BEB. ${ }^{10}$ Similarly host susceptibility may also be relevant in some cases of HFS, supported by observations that HFS is much more prevalent in Orientals, neurovascular compression of the facial nerve may not always result in HFS, ${ }^{5}$ and HFS can be inherited..$^{15}$ It would be interesting to determine whether BEB symptoms in our patients resolve after decompression surgery for HFS.

The trigemino-facial connections responsible for blinking involve afferent and efferent pathways in the pons and medulla oblongata. It is recognised that the blink reflex can be influenced by the cortex and basal ganglia. Activity dependent interaction between hand and face representations in the motor cortex before and after botulinum toxin injections in HFS patients suggest the presence of cortical plasticity in HFS. ${ }^{16}$ Impairment of the inhibitory trigeminofacial reflexes in HFS, and also excitatory trigemino-facial responses in the perioral muscles suggest a central control of these reflexes. ${ }^{17}$ There was a clinical report of two patients with coexistent movement disorders such as tremor, BEB, and HFS associated with caudate infarcts. ${ }^{18}$ These observations suggest that it is possible that altered cortical or subcortical processes in some HFS patients may modulate the blink response, resulting in BEB symptoms.

In a rat model of $\mathrm{BEB}$, reduced inhibition within the trigeminal blink circuits with weakening of the orbicularis oculi muscle triggers spasms of lid closure, similar to BEB in humans. ${ }^{10}{ }^{19}$ The observation of patients with facial palsy induced BEB suggests altered afferent input from facial weakness could cause an increase in the excitability of reflex blinking. ${ }^{10} 20$ Mild facial asymmetry and weakness is frequently observed in patients with chronic HFS with or without botulinum treatment. It is conceivable that facial weakness predisposed a few at-risk patients to develop BEB symptoms, as most of our study patients developed BEB after botulinum treatment for their HFS. In addition, one patient had Bell's palsy associated HFS and BEB.

In conclusion, we found a higher than expected prevalence of BEB in HFS patients compared with age and sex matched controls, suggesting that these two conditions may share overlapping pathophysiological mechanisms. Functional electrophysiological and imaging studies will be useful to further examine and clarify this association.

\section{Authors' affiliations}

E-K Tan, Department of Neurology, Singapore General Hospital, Republic of Singapore

L-L Chan, Department of Diagnostic Radiology, Singapore General Hospital, Republic of Singapore

K-K Koh, Healthway Medical Group 
Correspondence to: Dr E-K Tan, Department of Neurology, Singapore General Hospital, Outram road, Singapore 169608, Republic of Singapore; gnrtek@sgh.com.sg

Received 22 May 2003

In revised form 30 June 2003

Accepted 9 July 2003

\section{REFERENCES}

1 Digre K, Corbett JJ. Hemifacial spasm: Differential diagnosis, mechanism and treatment. Adv Neurol 1988;49:151-76.

2 Wang A, Jankovic J. Hemifacial spasm: clinical findings and treatment. Muscle Nerve 1998;12:1740-7.

3 Ehni G, Woltman HW. Hemifacial spasm. Review of 106 cases. Arch Neurol Psychiatry (Chicago) 1945;53:205-11.

4 Tan EK, Jankovic J. Bilateral hemifacial spasm: a report of five cases and a literature review. Mov Disord 1999;14:345-9.

5 Tan EK, Chan LL, Lim SH, et al. Role of magnetic resonance imaging and magnetic resonance angiography in patients with hemifacial spasm. Ann Acad Med Singapore 1999;28:169-73.

6 Sanders DB. Ephaptic transmission in hemifacial spasm: a single fiber EMG study. Muscle Nerve 1989;12:690-4.

7 Ishikawa M, Ohira T, Namiki J, et al. Abnormal muscle response (lateral spread) and F-wave in patients with hemifacial spasm. J Neurol Sci 1996;137:109-16

8 Grandas F, Elston J, Quinn N, et al. Blepharospasm: a review of 264 patients. J Neurol Neurosurg Psychiatry 1988;51:767-72.
9 Jankovic J. Etiology and differential diagnosis of blepharospasm and oromandibular dystonia. Adv Neurol 1988;49:103-16.

10 Hallett M. Blepharospasm: recent advances. Neurology 2002;59:1306-12.

11 Jankovic J, Ford J. Blepharospasm and orofacial-cervical dystonia: clinical and pharmacological findings in 100 patients. Ann Neurol 1983;13:402-11.

12 Jankovic J, Patel SC. Blepharospasm associated with brainstem lesions. Neurology 1983;33:1237-40.

13 Defazio G, Berardelli A, Abbruzzese G, et al. Risk factors for spread of primary adult onset blepharospasm: a multicentre investigation of the Italian movement disorders study group. J Neurol Neurosurg Psychiatry 1999;67:613-19

14 Misbahuddin A, Placzek MR, Chaudhuri KR, et al. A polymorphism in the dopamine receptor DRD5 is associated with blepharospasm. Neurology 2002;58:124-6.

15 Carter JB, Patrinely JR, Jankovic J, et al. Familial hemifacial spasm. Arch Ophthalmol 1990;108:249-50.

16 Liepert J, Oreja-Guevara C, Cohen LG, et al. Plasticity of cortical hand muscle representation in patients with hemifacial spasm. Neurosci Lett 1999;272:33-6.

17 Pavesi G, Cattaneo L, Chierici E, et al. Trigemino-facial inhibitory reflexes in idiopathic hemifacial spasm. Mov Disord 2003;18:587-92.

18 Arunabh, Jain S, Maheshwari MC. Blepharospasm hemifacial spasm and tremors possibly due to isolated caudate nucleus lesions. J Assoc Physicians India 1992;40:687-9.

19 Schicatano EJ, Basso MA, Evinger C. Animal model explains the origins of the cranial dystonia benign essential blepharospasm. J Neurophysiol 1997:77:2842-6.

20 Baker RS, Sun WS, Hasan SA, et al. Maladaptive neural compensatory mechanisms in Bell's palsy-induced blepharospasm. Neurology 1997;49:223-9. 